\section{In vitro antibiotic susceptibility of preoperative normal conjunctival bacteria}

CN Ta ${ }^{1}, \mathrm{~L} \mathrm{He}^{1}$ and $\mathrm{H}$ Mino de Kaspar ${ }^{1,2}$

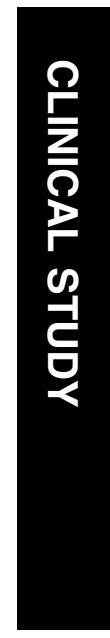

\author{
Abstract \\ Purpose To determine the antibiotic \\ susceptibility of preoperative conjunctival \\ bacterial flora. \\ Methods Antibiotic susceptibility of \\ conjunctival bacterial strains isolated from 142 \\ patients undergoing intraocular surgery was \\ determined using the Kirby-Bauer disc \\ diffusion technique. $\chi^{2}$ statistical analysis was \\ performed.
}

Results Among the $\mathbf{1 1 6}$ bacteria isolated, $95(82 \%)$ were coagulase-negative staphylococci (CNS). Most ( $>75 \%$ ) of them were susceptible to vancomycin, minocycline, aminoglycosides, gatifloxacin, levofloxacin, and moxifloxacin. Approximately one-half of CNS was resistant to erythromycin and azithromycin. Over $90 \%$ of streptococci were susceptible to levofloxacin, moxifloxacin, and gatifloxacin.

Conclusions Fluoroquinolones, specifically gatifloxacin, levofloxacin, and moxifloxacin continue to provide broad-spectrum coverage against common conjunctival bacterial flora.

Eye (2009) 23, 559-560; doi:10.1038/eye.2008.65; published online 21 March 2008

Keywords: antibiotics; bacteria; conjunctiva; fluoroquinolones; susceptibility

\section{Introduction}

Topical antibiotics are commonly prescribed in the preoperative period to possibly reduce the risk of infections following intraocular surgery. ${ }^{1}$ The purpose of this study is to provide an update on antibiotic susceptibility of normal ocular surface bacterial flora isolated from patients undergoing anterior segment intraocular surgeries.
Baseline conjunctival cultures were obtained from the operated eyes of patients who underwent anterior segment intraocular surgery at Stanford University from February 2004 to January 2005. The Kirby-Bauer disc diffusion technique was conducted in accordance with the guidelines of National Committee for Clinical Laboratory Standards (NCCLS). Statistical analysis was performed using $\chi^{2}$ test with StatsDirect software program (StatsDirect Software, Cheshire, UK).

\section{Results}

A total of 95 strains of coagulase-negative Staphylococcus (CNS), 13 Streptococcus species, 6 Staphylococcus aureus (S. aureus), 4

Propionibacterium acnes, and 3 Gram-negative rods (none were Pseudomonas) were isolated from 142 eyes of 142 patients.

The results of the antibiotic susceptibility tests for the 95 CNS strains are summarized in Figure 1. Among the commercially available antibiotics, the aminoglycosides and broad-spectrum fluoroquinolones have the highest susceptibility rates, whereas only about $50 \%$ of CNS were susceptible to the macrolides, such as erythromycin and azithromycin.

Antibiotic susceptibility results for Streptococcus species are summarized in Figure 2. All were susceptible to vancomycin. Among the fluoroquinolones, there were significant differences $(P=0.0048)$ between ciprofloxacin and ofloxacin ( $31 \%$ susceptibility) compared with levofloxacin, gatifloxacin, and moxifloxacin ( $92 \%$ susceptibility). Up to twothird of the streptococci were resistant to the aminoglycosides and macrolides.

None of the six $S$. aureus isolated were resistant to the fluoroquinolones or aminoglycosides except neomycin. One out of six was resistant to erythromycin and azithromycin.
Materials and methods
'Department of Ophthalmology, School of Medicine, Stanford University, Stanford, CA, USA

${ }^{2}$ Department of Ophthalmology, LudwigMaximilians-University, Munich, Germany

Correspondence: $\mathrm{CN} \mathrm{Ta}$, Department of Ophthalmology, Stanford University School of Medicine, 900 Blake Wilbur Drive, Room W-3036, Stanford, CA 94305, USA

Tel: +6507249981 ;

Fax: + 6504984222

E-mail: cta@

stanford.edu

Received: 11 December 2007

Accepted in revised form:

11 February 2008

Published online: 21 March 2008

The authors have no proprietary interest in any of the products used in this study. 


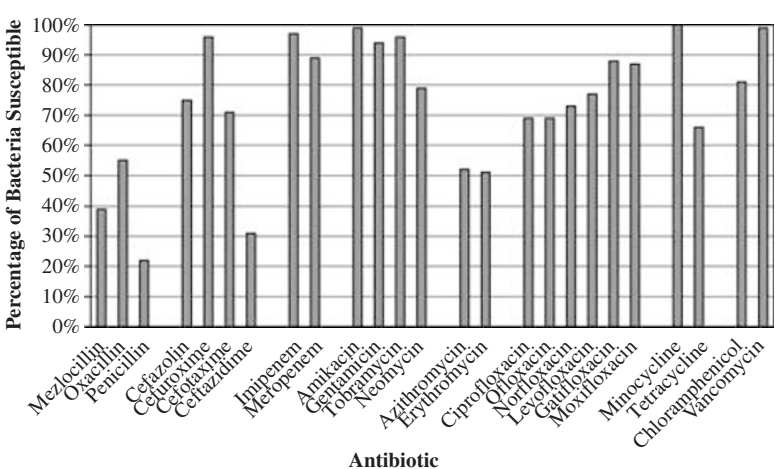

Figure 1 Susceptibility pattern of coagulase-negative Staphylococcus $(n=95)$.

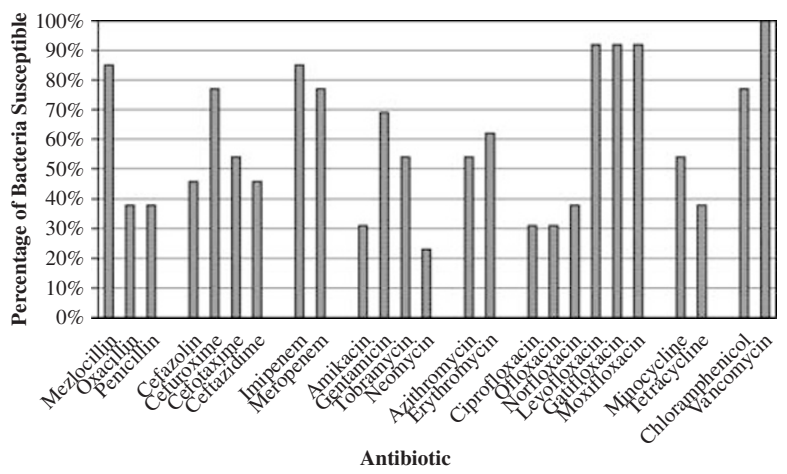

Figure 2 Susceptibility pattern of Streptococcus species $(n=13)$.

\section{Discussion}

The results of the current study are similar to the published results from Brazil, Europe, and the United States. ${ }^{2-4}$ In contrast, Harper et al demonstrated high resistance to fluoroquinolones among CNS isolated from patients with endophthalmitis. In our study, among the commercially available antibiotics, aminoglycosides and broad-spectrum fluoroquinolones have the highest susceptibility rate for all CNS isolated. Streptococci are commonly resistant to ciprofloxacin and ofloxacin, but highly susceptible to gatifloxacin, levofloxacin, and moxifloxacin. The broad-spectrum fluoroquinolones, specifically gatifloxacin, levofloxacin, and moxifloxacin continue to provide broad-spectrum coverage against common ocular bacterial flora in patients undergoing ocular surgery.

\section{Acknowledgements}

We thank the Hannelore-Georg Zimmermann Foundation, Munich, Germany; Edward E Hills Foundation, San Francisco,CA; Alcon Inc., Fort Worth, TX for their support of the research.

\section{References}

1 Liesegang TJ. Use of antimicrobials to prevent postoperative infection in patients with cataracts. Curr Opin Ophthalmol 2001; 12: 68-74.

2 Chalita MR, Hofling-Lima AL, Paranhos Jr A, Schor P, Belfort Jr R. Shifting trends in in vitro antibiotic susceptibilities for common ocular isolates during a period of 15 years. Am J Ophthalmol 2004; 137: 43-51.

3 Mino de Kaspar H, Koss MJ, He L, Blumenkranz MS, Ta CN. Antibiotic susceptibility of preoperative normal conjunctival bacteria. Am J Ophthalmol 2005; 139: 730-733.

4 Morrissey I, Burnett R, Viljoen L, Robbins M. Surveillance of the susceptibility of ocular bacterial pathogens to the fluoroquinolone gatifloxacin and other antimicrobials in Europe during 2001/2002. J Infect 2004; 49: 109-114.

5 Harper T, Miller D, Flynn Jr HW. In vitro efficacy and pharmacodynamic indices for antibiotics against coagulasenegative staphylococcus endophthalmitis isolates. Ophthalmology 2007; 114: 871-875. 